\title{
ANÁlISE DA EFICIÊNCIA TÉCNICA E DA PRODUTIVIDADE DOS SERVIÇOS DE ÁGUA E ESGOTOS NO BRASIL, 2006 A 2013.
}

Felipe Ponciano da Cruz* Ronaldo Seroa da Motta**

Alexandre Marinho***

\section{Resumo}

O marco regulatório da Lei Nacional do Saneamento Básico introduz mudanças fundamentais na prestação dos serviços de abastecimento de água, coleta e tratamento de esgotos. O objetivo do trabalho é estudar a eficiência do setor após a essa lei utilizando o método Data Envelopment Analysis em uma amostra contendo as 27 prestadoras representativas de cada ente da federação entre os anos de 2006 e 2013. Nossos resultados na análise estática indicam escores de eficiência baixos com grande disparidade entre as regiões do país. Na análise dinâmica observamos avanço na eficiência, em particular, entre os anos de 2010 e 2013, liderado pela expansão da fronteira.

Palavras-chaves: Setor de saneamento no Brasil; Eficiência; Análise de envoltória de dados.

\section{ANALYSIS OF TECHNICAL EFFICIENCY AND PRODUCTIVITY OF WATER AND SEWAGE SERVICES IN BRAZIL, 2006 TO 2013.}

\begin{abstract}
The regulatory framework of the National Basic Sanitation Law introduces fundamental changes in the provision of water supply, sewage collection and treatment services. The objective of the paper is to study the efficiency of the sector after this law applying the Data Envelopment Analysis method in a sample with 27 representative providers of each entity of the federation between the years of 2006 and 2013. Our results for the static analysis indicate low efficiency scores with great disparity between the regions. The dynamic analysis showed increasing efficiency levels, mainly between the years of 2010 and 2013 , led by the expansion of the frontier.
\end{abstract}

Key-Words: Sanitation sector in Brazil; Efficiency; Data envelopment analysis

\section{Área: Crescimento, Competitividade e Produtividade}

\section{Classificação de assunto: JEL:L9}

\footnotetext{
*Mestre em Economia pela UERJ. Contato: f1ponciano@gmail.com

** Professor Doutor em PPGCE/UERJ. Contato: seroadamotta.ronaldo@ gmail.com

*** Professor Doutor em PPGCE/UERJ. Contato: alexmarinho1356@gmail.com
} 


\section{INTRODUÇÃO}

Os serviços de distribuição de água potável e coleta e tratamento de esgoto influenciam tanto a saúde da população quanto a qualidade do meio ambiente e, portanto, são fundamentais para redução da pobreza e para o desenvolvimento sustentável. Desde a captação da água bruta até o descarte dos esgotos, os serviços de água e esgotos dependem e interferem nas condições ambientais. Consequentemente, diversas doenças são influenciadas pelas condições inadequadas desses sistemas, tais como, diarreias, febres entéricas, hepatite A, esquistossomose, leptospirose, teníases, micoses e conjuntivites (Mendonça e Seroa da Motta, 2007 e Brasil, 2002).

A evolução do setor de água e esgotos no Brasil foi extremamente influenciada pelo Plano Nacional de Saneamento Básico (PLANASA), implementado em 1969 durante o regime militar, e extinto no início da década de 90. O programa era fomentado pelo BNH (Banco Nacional de Habitação) e operado pelas Companhias Estaduais de Saneamento Básico (CESBs) (Salles, 2008 e Seroa da Motta, 2005).

O PLANASA aumentou bastante a cobertura de saneamento no país, mas, apesar de ter sido bastante efetivo quanto ao abastecimento de água potável, deixou de lado a coleta de esgoto. Ainda hoje, mais de duas décadas após o fim do programa, observamos a predominância das CESBs e a atuação deficiente no serviço de coleta e tratamento de esgoto em relação à distribuição de água potável.

Com o fim do PLANASA e do Sistema Financeiro do Saneamento (SFS), houve um vazio regulatório e institucional. Desse modo, as décadas de 1990 e 2000 foram marcadas por intensos debates sobre o novo marco regulatório para o saneamento, o qual finalmente só implementado em 2010 com a regulamentação da lei da Política Nacional Saneamento Básico (LNSB). ${ }^{1}$

A LNSB define que os serviços de saneamento ${ }^{2}$ serão prestados com eficiência e sustentabilidade econômica e os contratos de concessão devem prever metas progressivas e graduais de expansão de serviços, de qualidade, de eficiência e de uso racional da água e da energia. E mais ainda que essa prestação deve conter mecanismos e procedimentos para a avaliação sistemática da eficiência e eficácia das ações programadas. A política tarifária deve, portanto, incluir incentivos à eficiência na prestação de serviços.

Há também na LNSB uma complexa atribuição de competências entre os entes federados ${ }^{3}$. Os Municípios são os titulares dos serviços de saneamento e podem executá-los diretamente ou por meio de concessão. É de competência municipal a organização, regulação e fiscalização da prestação de tais serviços, bem como a elaboração do Plano Municipal de Saneamento Básico (PMSB). Assim, os Municípios podem instituir suas próprias agências reguladoras ou delegar a responsabilidade à entidade reguladora de seu Estado. No caso da operação dos serviços do município ser realizada por uma operadora estadual (CESB), a regulação é de responsabilidade de uma agência estadual.

Em suma, a LNSB define o Município como titular das quatro funções essenciais para a coordenação dos serviços de saneamento: prestação dos serviços, regulação, físcalização e planejamento. A única função que não delegável é a do planejamento representada pelo PMSB mas compartilham as outras funções regulatórias com os municípios em regiões metropolitanas e microrregiões.

A gestão dos recursos hídricos que concede outorga para captação de água e o despejo de esgotos e determina a qualidade das águas é regulada pela Política Nacional dos Recursos Hídricos com competência federal para os cursos d’águas que banham mais de um Estado ou país ou dos estados nos casos que se limitam ao seu território.

Muito se avançou na cobertura dos serviços, embora 18,3\% das crianças de 0 a 14 anos de idade residentes em domicílios particulares permanentes não possuam acesso à rede geral de

\footnotetext{
${ }^{1}$ Lei no $11.445 / 07$ regulamentada pelo decreto $n^{\circ}=7.217$ de 21 de junho de 2010 .

2 Brasil (2007)

${ }^{3}$ Inclusive a definição dessas atribuições foi um dos principais obstáculos na elaboração do marco regulatório e ainda é fonte de litígio entre estados e municípios, ver Estache, Garsous e Seroa da Motta (2016).
} 
abastecimento de água e apenas 55,5\% tenham têm esgotamento sanitário de rede geral ou fossa séptica e somente 39\% dos esgotos do país são tratados (IBGE, 2014 e Brasil, 2015).

Logo continua importante observar como tem evoluído a eficiência do setor. Inúmeros estudos nas últimas duas décadas tentaram explicar a evolução da eficiência das operadoras dos serviços de saneamento no Brasil. Esses estudos analisam o desempenho dessas empresas e, em muitos casos, controlando pelo efeito da natureza da propriedade (privada versus pública) e da jurisdição da operação (regional versus local).

Nesses estudos se observa que há dominância do método Data Envelopment Analysis (DEA) em grande parte devido a sua flexibilidade na função de produção e no tratamento da característica intrínseca de multiproduto do setor.

No Brasil, estudos da eficiência utilizando a DEA aplicados ao fornecimento de água e esgoto tiveram início na década de 2000 e, além dos temas sobre a influência da propriedade e da regulação na eficiência, também existem análises relativas à área de abrangência, ou seja, se é mais eficiente ter uma empresa local ou regional. Nos trabalhos brasileiros também é mais comum a análise conjunta do setor de água e esgoto que, em geral, são serviços prestados por uma mesma companhia.

Carmo e Távora Junior (2003), utilizando dados do ano de 2000, calcularam a eficiência técnica e de escala de 26 Companhias Estaduais de Saneamento Básico com um modelo output orientado. Como respostas obtiveram que a eficiência técnica é superior à eficiência de escala e que o setor apresenta retornos crescentes de escala.

Tupper e Resende (2004) por meio de um modelo com retornos variáveis de escala e output orientado, com dados de 1996 a 2000, mensurou a eficiência de 20 Companhias Estaduais de Saneamento. Como resultados encontraram um desempenho abaixo do ótimo e verificaram a possibilidade de diminuir as diferenças entre as regiões de modo a realizar uma melhor comparação de eficiência.

Um dos dois trabalhos brasileiros a utilizar o Índice de Malmquist é o de Seroa da Motta e Moreira (2006). Foram analisadas 104 Companhias Brasileiras de Saneamento, abrangendo tanto as regionais quanto as locais, entre os anos de 1998 e 2002. Por meio de um modelo output orientado foram mensuradas as eficiências de escala e técnica. Como resultado, não foi verificada diferença significativa entre as prestadoras públicas e privadas e, em relação ao Índice de Malmquist, o efeito emparelhamento foi superior ao efeito mudança de fronteira e as prestadoras privadas chegaram mais depressa na fronteira em relação às públicas.

Sampaio e Sampaio (2007) analisaram 36 Companhias de Saneamento brasileiras entre 1998 e 2003 por meio de um modelo com retornos variáveis de escala e output orientado. Os autores encontraram retornos crescentes de escala e aferiram que os serviços relativos à distribuição de água são mais eficientes do que os relativos aos esgotos.

Utilizando os dados de 57 Companhias de Saneamento paulistas entre os anos de 1995 e 2004, Grigolin (2007) utilizou modelos output orientados com retornos constantes e variáveis de escala. Como resultado, encontrou na maior parte das simulações retornos decrescentes de escala. No modelo com retornos constantes de escala as prestadoras privadas foram mais eficientes que as regionais. Com retornos variáveis de escala as empresas regionais apresentaram escores maiores de eficiência.

Sato (2011) analisou a eficiência de 26 companhias de saneamento das capitais dos Estados brasileiros com dados entre os anos de 2005 e 2008. Foram utilizados modelos com retornos constantes e modelos com retornos variáveis de escala, e o autor verificou grande variabilidade de eficiência entre as regiões do país bem como espaço para melhoria de eficiência no setor.

Já Barbosa (2012) procurou explicar a eficiência por meio da estrutura regulatória com uma amostra de 41 prestadoras de serviço de água e esgoto. Neste trabalho foi utilizado o DEA, input orientado, com o modelo aditivo Dynamic Slack Based Model - DSBM para determinar o escore de eficiência e posteriormente foi utilizada a técnica de regressão Generalized Estimating EquationGEE. O autor verificou que as prestadoras não estão ganhando produtividade ao longo do tempo e 
que as performances não são explicadas por experiência, âmbito de atuação e a especialização das agências reguladoras.

O segundo trabalho que utilizou as prestadoras brasileiras que utilizou o Índice de Malmquist é o de Carvalho (2014). A autora utilizou modelos output orientados para mensurar a eficiência de 29 prestadoras entre os anos de 2006 e 2011. O Índice de Malmquist revelou uma queda na produtividade entre os anos apresentados, e vale observar que as prestadoras que passaram a ser reguladas por agência após a LNSB, 2007, apresentaram maiores avanços relativos.

Literatura similar internacional existe. Lin e Berg (2008) utilizaram as seguintes técnicas para avaliar a eficiência de 38 prestadoras de serviço de distribuição de água peruanas entre os anos de 1998 e 2001: i) modelo DEA básico; ii) modelo DEA com estrutura de preferências; e iii) Índice de Malmquist com a qualidade incorporada. Como resultado os autores encontraram uma pequena melhora na qualidade dos serviços entre os anos analisados, bem como os impactos provocados pela introdução de aspectos de qualidade na análise de eficiência.

Byrnes et al (2010) analisou os efeitos de diversas ações políticas na eficiência de 52 prestadoras de serviços de água nas regiões australianas de New South Wales e Victoria. Para tanto utilizou modelos input orientados com retornos constantes e com retornos variáveis de escala, além do Índice de Malmquist. Como resultado, o trabalho verificou grande influência das políticas urbanas de água na dinâmica da eficiência.

Portela et al (2011) utilizou o Índice de meta-Malmquist circular para analisar a mudança na produtividade de 22 prestadoras de serviços de distribuição de água da Inglaterra e do Reino Unido entre os anos de 1993 e 2007. O Índice meta-Malmquist verifica a mudança de produtividade em referência a uma meta-fronteira de modo a permitir comparar ao longo do tempo as performances das companhias. Como resultado houve melhoria de eficiência entre os anos de 1993 e 2005, mas depois desse ano a tendência foi de queda até 2007.

Também na Austrália, Abbott, Cohen e Wang (2012) analisaram a performance dos setores de água e esgotos das cidades de Sydney, Melbourne, Brisbane, Adelaide, Perth e Canberra. Para tanto utilizaram o Índice de Malmquist com dados entre os anos de 1995 e 2008. Como resultado, encontraram incrementos positivos de produtividade nos centros urbanos maiores, independente da estrutura de sua indústria. $O$ trabalho também ressalta a influência de fatores imprevisíveis e incontroláveis na eficiência.

O presente trabalho analisa a eficiência técnica das prestadoras estaduais (CESBs) com o método Data Envelopment Analysis (DEA) e o Índice de Malmquist para sua evolução ao longo do tempo, ambos em conjunto com a técnica bootstrap. A grande vantagem de utilizar o bootstrap nesses dois casos é acrescentar intervalos de confiança a métodos anteriormente determinísticos.

Vale ressaltar que não foi encontrado na literatura brasileira sobre a eficiência do setor de saneamento outro estudo que utilizasse as técnicas bootstrap associados a técnicas de DEA de modo a encontrar escores mais robustos, nem a utilização do Índice Malmquist com auxílio do bootstrap de modo a encontrar intervalos de confiança para a análise dinâmica.

O trabalho se divide em mais quatro seções além desta introdução: metodologia; modelo e dados; resultados; e conclusão.

\section{METODOLOGIA}

A eficiência econômica é entendida como o máximo de outputs que a empresa pode produzir dada quantidade de inputs utilizada, ou, da mesma maneira, como o mínimo de inputs que podem produzir uma dada quantidade de outputs, ou seja, os valores ótimos de outputs e inputs utilizados.

Marinho e Façanha (2001), ao estudarem os problemas de avaliação em programas sociais, relacionaram efetividade "[...] à implementação e ao aprimoramento de objetivos, independentemente das insuficiências de orientação e das falhas de especificação rigorosa dos objetivos iniciais declarados do programa" (Marinho e Façanha, 2001, p. 6). Dessa maneira o ideal seria avaliarmos efetividade e não eficiência.

Podemos sumarizar este raciocínio conforme Bogetoft e Otto (2011). Em um modelo racional ideal temos uma entidade econômica em busca dos melhores meios para atingir seus objetivos e, 
dessa forma, podemos assumir que uma firma transforma $m$ inputs $\mathrm{x}^{*} \in \mathrm{R}^{\mathrm{m}}$ em $\mathrm{n}$ outputs $\mathrm{y}^{*} \in \mathrm{R}^{\mathrm{n}} \mathrm{e}$ que a função objetivo ou função de utilidade é dada por: $U: R^{m+n} \rightarrow R$, onde $U(x, y)$ é a utilidade ligada ao plano de produção $(\mathrm{x}, \mathrm{y})$. Devemos assumir também que o conjunto de vetores $(\mathrm{x}, \mathrm{y})$ possíveis é $\mathrm{T} \subseteq \mathrm{R}^{\mathrm{m}+\mathrm{n}}$.

Sendo assim, o vetor $\left(\mathrm{x}^{*}, \mathrm{y}^{*}\right)$ é ótimo se resolve o problema de decisão básico abaixo se, e somente se, a firma realizar o melhor e mais efetivo uso do seu potencial:

máx $U(x, y)$

sujeito a: $(x, y) \in \mathrm{T}$

Entretanto, dificilmente encontramos na prática as condições ideais para realizarmos avaliações de efetividade, pois desconhecemos o plano de produção factível, T, e/ou o critério de avaliação agregado, U(.). Outro problema apontado é que os múltiplos outputs são de difícil agregação.

A proposta da moderna teoria da análise de produtividade é focar em eficiência no lugar da efetividade, de modo a construir uma tecnologia usando a análise da eficiência relativa. Podemos sumarizar esse raciocínio da seguinte maneira: $\left(\mathrm{x}^{\prime}, \mathrm{y}^{\prime}\right) \in \mathrm{T} \subseteq \mathrm{R}^{\mathrm{m}+\mathrm{n}}$ é eficiente relativamente à tecnologia $\mathrm{T}$ se e somente se para todo $(\mathrm{x}, \mathrm{y}) \in \mathrm{T}: \mathrm{x} \leq \mathrm{x}^{\prime} ; \mathrm{y} \geq \mathrm{y}^{\prime} \rightarrow \mathrm{x}=\mathrm{x}^{\prime}$ e $\mathrm{y}=\mathrm{y}^{\prime}$, e, portanto, não podemos encontrar melhor vetor do que $\left(\mathrm{x}^{\prime}, \mathrm{y}^{\prime}\right)$.

Assim percebemos que a eficiência é uma condição necessária, embora não suficiente para efetividade, e utilizamos eficiência invés de efetividade devido ao desconhecimento de U(.)e da real tecnologia $\mathrm{T}$, de modo a encontrar o conjunto de planos eficientes, $\mathrm{T}^{\mathrm{E}}$, que é o "menor conjunto suficiente de alternativas a considerar" na busca da efetividade (Bogetoft e Otto, 2011, p. 53).

Nesse estudo avaliamos a eficiência estática e a dinâmica.

\subsection{Análise Estática}

Usaremos a metodologia Data Envelopment Analysis (DEA) que é uma metodologia não paramétrica (não propõe a estimação de uma função com parâmetros) e não estocástica (não é associado a qualquer distribuição de probabilidade). A fronteira de produção é gerada implicitamente via programação linear.

Essa escolha se deve à sua praticidade de não necessitar de pré-especificações sobre a função de produção com maior flexibilidade no tratamento de multiproduto e na análise dinâmica da eficiência. Além disso, os preços no setor de saneamento muitas vezes são decididos politicamente de maneira que a influência das empresas monopolísticas é grande. Como a DEA não necessita da especificação de preços para calcular a eficiência técnica, ela é recomendada para setores com preços deturpados.

Para atenuar a desvantagem dessa abordagem não-paramétrica utilizarmos a técnica bootstrap, a partir de Simar e Wilson (1998), para corrigir o viés dos escores e estimar intervalos de confiança.

As DMU's (Decison Making Units) são os elementos básicos das análises de eficiência. Elas devem ser homogêneas, utilizar os mesmos inputs e outputs, bem como apresentarem autonomia na tomada de decisão.

A eficiência de cada DMU é medida pela otimização da soma ponderada das saídas (outputs) dividida pela soma ponderada das entradas (inputs), ou seja, pela combinação entre output virtual e input virtual de modo a maximizar $\frac{\text { output virtual }}{\text { input virtual }}$,ou, de maneira equivalente, minimizar $\frac{\text { input virtual }}{\text { output virtual }}$.

Por definição não podemos ter o input virtual maior do que o output virtual e, portanto, o output virtual deve ser menor ou igual que o input virtual. Isto posto, uma DMU plenamente eficiente é aquela que consegue transformar todo o input virtual em output virtual e assim temos $\frac{\text { input virtual }}{\text { output virtual }}=1$. 
Os modelos de DEA admitem diferentes naturezas de retornos de escala: i) o modelo $\mathrm{CCR}^{4}$ (Charnes, Cooper e Rhodes) que impõe retornos constantes de escala; e ii) o modelo BCC $^{5}$ (Banker, Charnes e Cooper) que admite retornos variáveis de escala.

Podemos também escolher a orientação do Modelo de DEA, entre input ou output orientado. Um modelo output orientado maximiza os outputs mantendo os inputs inalterados, enquanto que um modelo input orientado minimiza os inputs mantendo os outputs inalterados.

O modelo (2) apresenta o Modelo da Envoltória, output orientado, e com retornos variáveis de escala. Onde: $\mathrm{h} \geq 1$, é a medida de expansão radial, quando $\mathrm{h}_{0}=1$ dizemos que a DMU é eficiente, $\lambda$ 's são os pesos das combinações convexas das DMU's e $x_{i k}$ e $y_{j k}$ são os inputs i e outputs $\mathrm{j}$ da DMU k, $\mathrm{k}=1, \ldots, \mathrm{n} ; \mathrm{x}_{i o}$ e $y_{j o}$ são os inputs i e outputs $\mathrm{j}$ da DMU 0. A DMU 0 é a DMU sob análise e o problema será resolvido n vezes, uma vez para cada DMU.

A eficiência é representada pela função objetivo, o qual é o valor que devemos multiplicar todos os outputs para colocamos as DMU sobre a fronteira eficiente. Com as duas restrições garantimos que cada aumento dos outputs não chegue a ultrapassar a fronteira definida pelas DMUs eficientes e também que não modificaremos o atual nível dos inputs da DMU.

Máx $h_{0}$

sujeito a:

$-h_{0} y_{j 0}+\sum_{k=1}^{n} y_{j k} \lambda_{k} \geq 0, \forall \mathrm{j}$

$x_{i 0}-\sum_{k=1}^{n} x_{i k} \lambda_{k} \geq 0, \forall \mathrm{i}$

$\sum_{k=1}^{n} \lambda_{k}=1$

$\lambda_{k} \geq 0, \forall \mathrm{k}$

Entretanto, conforme Simar e Wilson (1998), o bootstrap normal não se aplica ao método DEA. Não poderíamos reamostrar diretamente o conjunto de escores do DEA, pois dessa maneira teríamos que admitir que os escores sejam independentes e identicamente distribuídos, o que iria contra a suposição que eles dependem da relação entre inputs e outputs. Tampouco poderíamos reamostrar simplesmente os inputs (ou outputs) para estimar a tecnologia e a eficiência de determinada DMU, pois poderíamos encontrar escores maiores do que a unidade, ou seja, vetores que não pertencem à tecnologia. Tal ocorrência é mais provável em DMUs mais próximas à fronteira original.

Os escores do DEA também apresentam o problema de serem bastante concentrados em torno do valor unitário. Para enfrentar esta dificuldade Simar e Wilson (1998) propõem os métodos de alisamento da distribuição e de reflexão, bem como a correção do viés.

$\mathrm{O}$ alisamento consiste em corrigir a amostra gerada pelo bootstrap com a utilização do fator h $\epsilon$, onde h é a faixa de amplitude (bandwidth) e $\in$ é gerado a partir de uma distribuição normal. O alisamento visa evitar o surgimento de muitos valores repetidos, que gerariam picos na distribuição do estimador. Por meio do método da reflexão evitamos o problema do surgimento de muitos escores de eficiência próximos da unidade, o que introduziria viés e inconsistência no estimador. A cada escore de eficiência $\widetilde{E}_{k}$ é calculado um reflexo $2-\widetilde{E}_{k}$, e usamos sempre o valor menor ou igual a unidade. Assim, a média será $\left[\left(\widetilde{E}_{k}+2-\widetilde{E}_{k}\right)\right] / 2=1$ e simétrica em torno da unidade.

\footnotetext{
${ }^{4}$ Ver Charnes, Cooper, Rhodes (1978).

${ }^{5}$ Ver Banker, Charnes, Cooper (1984)
} 
O escore de eficiência do DEA é benevolente, ou seja, superestima a eficiência real, isso ocorre devido à tecnologia $\widehat{T}$, gerada pelo modelo ser sempre o menor conjunto suficiente, pois algumas observações podem não constar da amostra. O estimador com o viés corrigido é dado por (Bogetoft e Otto, 2011, p. 173):

$\tilde{\theta}^{\mathrm{k}}=2 \widehat{\theta}^{\mathrm{k}}-\bar{\theta}^{\mathrm{k} *}$

Onde:

○ $\tilde{\theta}^{\mathrm{k}}$ é o estimador da eficiência $\theta^{\mathrm{k}}$ com o viés corrigido, onde $\theta^{\mathrm{k}}$ é a eficiência baseada na tecnologia verdadeira mas desconhecida $\mathrm{T}$.

- $\widehat{\theta}^{\mathrm{k}}$ é o estimador de eficiência obtido a partir da tecnologia T estimada pela DEA.

○ $\bar{\theta}^{\mathrm{k} *}$ é a média de $\theta^{\mathrm{k} *}$ que é a eficiência estimada por bootstrap de $\theta^{\mathrm{k}}$.

Podemos sumarizar de maneira simplificada o bootstrap que iremos aplicar da seguinte maneira (Bogetoft e Otto, 2011, p. 173):

1. Computar $\hat{\theta}^{k}$ como soluções para $\min \left\{\theta \mid \theta\left(x^{k}, y^{k}\right) \in \widehat{T}\right\}$ para $k=1, \ldots, n$.

2. Usar o bootstrap utilizando a amostra alisada de $\widehat{\theta}^{1}, \ldots, \hat{\theta}^{k}$ para obter uma réplica $\theta^{1 *}, \ldots, \theta^{k *}$ do modo abaixo:

a. Usar o bootstrap na amostra com reposição a partir de $\hat{\theta}^{1}, \ldots, \hat{\theta}^{k}$, e chamar os resultados de $\beta^{1}, \ldots, \beta^{k}$.

b. Simular as variáveis independentes aleatórias $\epsilon^{1}, \ldots, \epsilon^{k}$ com distribuição normalpadrão.

c. Calcular (utilizando o alisamento e a reflexão):

$\tilde{\theta}^{\mathrm{k}}=\left\{\begin{array}{l}\beta^{\mathrm{k}}+\mathrm{h} \epsilon^{\mathrm{k}} \operatorname{se} \beta^{\mathrm{k}}+\mathrm{h} \epsilon^{\mathrm{k}} \leq 1 \\ 2-\beta^{\mathrm{k}}-\mathrm{h} \epsilon^{\mathrm{k}} \text { caso contrário }\end{array}\right.$

Por construção $\tilde{\theta}^{\mathrm{k}} \leq 1$.

d. Ajustar $\tilde{\theta}^{k}$ para obter parâmetros com variância assintoticamente correta, e então estimar a variância $\hat{\sigma}^{2}=\frac{1}{n} \sum_{k=1}^{K}\left(\hat{\theta}^{k}-\overline{\hat{\theta}}\right)^{2}$ e calcular $\bar{\beta}=\frac{1}{n} \sum_{k=1}^{K} \beta^{k}$ :

$\theta^{\mathrm{k} *}=\bar{\beta}+\frac{1}{\sqrt{1+\mathrm{h}^{2} / \widehat{\sigma}^{2}}}\left(\hat{\theta}^{\mathrm{k}}-\bar{\beta}\right)$

3. Calcular o bootstrapped input $x^{k b}=\frac{\widehat{\theta}^{k}}{\theta^{k *}} x^{k}$ baseado na eficiência calculada no bootstrap.

4. Solucionar a o problema de programação da DEA para estimar $\theta^{k b}$ como:

$\theta^{\mathrm{kb}}$

$=\min \left\{\theta \geq 0 \mid y^{k} \leq \sum_{j=1}^{K} \lambda_{j} y_{j}, \theta x^{k} \geq \sum_{j=1}^{K} \lambda_{j} x_{j}^{k b}, \lambda_{j} \geq 0, \sum_{j=1}^{K} \lambda_{j}=1\right\}$

$(\mathrm{k}=1, \ldots, \mathrm{n})$

5. Repetir os passos a partir de (2.a) para obter as estimativas bootstrap $\left(\theta^{1 b}, \ldots, \theta^{K b}\right)(b=$ $1, \ldots, B)$.

6. Calcular a média e variância de $\left(\theta^{1 b}, \ldots, \theta^{K b}\right)$ para conseguir a estimativa bootstrap $\theta^{k *}$, e o estimador com o viés corrigido $\tilde{\theta}^{k *}$, e a variância:

$\widehat{\sigma 2}=\frac{1}{\mathrm{~B}} \sum_{\mathrm{b}=1}^{\mathrm{B}}\left(\theta^{\mathrm{kb}}-\bar{\theta}^{\mathrm{k} *}\right)^{2}$ 
Ao final do processo a distribuição de $\left(\hat{\theta}^{k}-\theta\right)$ é desconhecida mas pode ser aproximada pela distribuição de $\left(\theta^{k *}-\hat{\theta}^{k}\right)$. $\theta$ é o escore de eficiência real, mas desconhecido, $\hat{\theta}^{k}$ é o escore de eficiência tradicional obtido na DEA e $\theta^{k *}$ é a estimativa do escore de eficiência da DEA obtida no bootstrap.

\subsection{Análise Dinâmica}

Para entendermos evolução dinâmica da eficiência utilizaremos o índice de Malmquist que representa a variação na produtividade total dos fatores entre os períodos $\mathrm{s}$ e $\mathrm{t}$ dessa forma (Bogetoft e Otto, 2011):

$M(s, t)=\sqrt{\frac{E(t, s) E(t, t)}{E(s, s) E(s, t)}}$

Onde:

- $E(t, s)$ é a eficiência da DMU medida no período t com tecnologia do período s;

- $E(t, t)$ é a eficiência da DMU medida no período t com tecnologia do período t;

- $E(s, t)$ é a eficiência da DMU medida no período s com tecnologia do período t;

- $E(s, s)$ é a eficiência da DMU medida no período s com tecnologia do período s;

Dessa forma, podemos tomar as seguintes conclusões sobre os valores do índice:

○ $M(s, t)>1$ : houve uma mudança positiva na produtividade total dos fatores entre os períodos $s$ e $t$

○ $M(s, t)=1$ : não houve qualquer mudança na produtividade total dos fatores entre os períodos $s$ e $t$

○ $M(s, t)<1$ : houve mudança negativa na produtividade total dos fatores entre os períodos $s$ e $t$.

Uma característica importante do índice de Malmquist é que ele pode ser decomposto em dois fatores:

$M(s, t)=\sqrt{\frac{E(t, s) E(s, s)}{E(t, t) E(s, t)}} \frac{E(t, t)}{E(s, s)}=T C(s, t) E C(s, t)$

Onde:

$T C(s, t)=\sqrt{\frac{E(t, s) E(s, s)}{E(t, t) E(s, t)}}$

$E C(s, t)=\frac{E(t, t)}{E(s, s)}$

Se tivermos $E(t, s)>E(t, t)$ podemos afirmar que a tecnologia progrediu, pois a mudança de tecnologia de $\mathrm{t}$ para $\mathrm{s}$ foi suficiente para aumentar a eficiência. A mesma lógica serve para $E(s, s)>E(s, t)$.

O índice de Mudança Tecnológica, $\operatorname{TC}(s, t)$, representa a média geométrica de dois índices, $E(t, s) / E(t, t)$ e $E(s, s) / E(s, t)$. Portanto, TC mede a mudança tecnológica e, se encontrarmos $T C(s, t)>1$, temos um progresso tecnológico, ou seja, uma mudança (expansão) da fronteira.

O índice de Mudança de Eficiência, EC $(s, t)$ ("catch up") mede a aproximação da firma da fronteira, efeito emparelhamento, caso $E(t, t)>E(s, s)$ teremos $E C(s, t)>1$ e sabemos que a firma se aproximou da fronteira. 
Podemos utilizar o bootstrap para encontrar intervalos de confiança dos resultados encontrados ao aplicar o índice de Malmquist (Simar e Wilson, 1999). Dessa maneira devido ao desconhecimento da distribuição verdadeira do índice de Malmquist o bootstrap é utilizado para estimar a distribuição e definir o intervalo de confiança seguindo os seguintes passos:

1) Estimar o índice de Malmquist $\widehat{M}_{\mathrm{t}, \mathrm{s}}\left(\mathrm{x}^{\mathrm{s}}, \mathrm{y}^{\mathrm{s}}, \mathrm{x}^{\mathrm{t}}, \mathrm{y}^{\mathrm{t}}\right)$ das K DMUs da maneira descrita acima;

2) Obter as pseudoamostras $\left(x^{t *}, y^{t *}\right)$ de cada DMU para construir a tecnologia via bootstrap com alisamento e reflexão;

3) Calcular, utilizando a amostra obtida no passo 2, a estimativa bootstrap do índice de Malmquist $\widehat{M}_{t, s}^{k b *}\left(\mathrm{x}^{\mathrm{s}}, \mathrm{y}^{\mathrm{s}}, \mathrm{x}^{\mathrm{t}}, \mathrm{y}^{\mathrm{t}}\right)$ de cada uma das $\mathrm{k}$ DMUs;

4) Repetir B vezes o passos 2 e 3, para obter um conjunto de estimativas $\widehat{M}_{t, s}^{k b *}\left(\mathrm{x}^{\mathrm{s}}, \mathrm{y}^{\mathrm{s}}, \mathrm{x}^{\mathrm{t}}, \mathrm{y}^{\mathrm{t}}\right)$;

5) Obtêm-se o intervalo de confiança a partir deste conjunto de estimativas.

Com o intervalo de confiança estaremos habilitados a verificar a significância do índice de Malmquist. Caso o intervalo de confiança encontrado tenha os dois extremos menores do que a unidade podemos afirmar que a produtividade decaiu, caso o intervalo de confiança tenha os dois extremos maiores do que a unidade podemos afirmar que a produtividade aumentou, enfim se o intervalo de confiança tiver um extremo menor do que a unidade e outro maior não podemos afirmar coisa alguma sobre o comportamento da produtividade entre os períodos em questão.

\section{MODELO E DADOS}

A decisão sobre o modelo a ser utilizado se baseou, principalmente, na revisão de literatura acima e no entendimento do funcionamento do setor e seus objetivos.

Em nosso modelo, as DMUs são as prestadoras de serviços de distribuição de água e coleta e tratamento de esgotos. Essa escolha é justificada pela decisão sobre a alocação de recursos a ser tomada pelas empresas. Pressupomos que as DMUs se defrontam com a mesma tecnologia (T), que é desconhecida, e utilizaremos DEA para estimar a tecnologia $(\widehat{T})$ com o uso da DEA e do índice de Malmquist.

O modelo será output orientado, pois as empresas são em sua maioria públicas onde o corte de gastos é dificultado e a demanda pelos serviços de fornecimento de água e de esgotamento sanitário não está plenamente atendida.

Quanto a escolha entre CCR (que impõe retornos constantes de escala) e o modelo BCC (que admite retornos variáveis de escala) optamos pelo de retornos variáveis de escala devido ao reconhecimento da existência de dificuldades para as prestadoras mudarem de tamanho no curto prazo, bem como a grande variação de dimensão entre as prestadoras analisadas.

Os dados que serão utilizados no trabalho serão da série histórica do SNIS, que possui dados das prestadoras de saneamento a partir de 1995, os quais estão disponíveis via internet ${ }^{6}$. Foram selecionadas como amostra 27 prestadoras, as maiores de cada Estado mais a do Distrito Federal, entre os anos de 2006 e 2013 totalizando 216 observações.

Os outputs $y_{k}=\left(\mathrm{y}_{1 k}, \ldots, \mathrm{y}_{5 k}\right)$ utilizados são listados abaixo, conforme a nomenclatura do SNIS:

- Item AG003 - Quantidade de Economias ${ }^{7}$ Ativas de Água - Unidade: economia.

- Item AG010 - Volume de Água Consumido - Unidade: $1.000 \mathrm{~m}^{3} / \mathrm{ano}$.

- Item ES003 - Quantidade de Economias Ativas de Esgoto - Unidade: economia.

- Item ES005 - Volume de Esgoto Coletado - Unidade: $1.000 \mathrm{~m}^{3} / \mathrm{ano}$.

- Item ES006 - Volume de Esgoto Tratado- Unidade: $1.000 \mathrm{~m}^{3} / \mathrm{ano}$.

Apesar da cobrança pela coleta e tratamento de esgoto serem feitas de maneira conjunta, decidimos por tratá-los como produtos separados a exemplo de Seroa e Moreira (2006).

\footnotetext{
${ }^{6}<$ http://www.snis.gov.br/>

${ }^{7}$ ECONOMIA - Imóvel de uma única ocupação, ou subdivisão de imóvel com ocupação independente das demais, perfeitamente identificável ou comprovável em função da finalidade de sua ocupação legal, dotado de instalação privativa ou comum para o uso dos serviços de abastecimento de água ou de coleta de esgoto. Ex.: um prédio com 10 apartamentos possui uma ligação 10 economias.

Fonte: $<$ http://www.mzweb.com.br/copasa/web/conteudo_pt.asp?idioma=0\&tipo=28109\&conta=28\&id=74922 $>$.
} 
Os outputs com os números de economias de água e esgoto (AG003 e ES003) são os mais utilizados na literatura e entendemos que eles captam o objetivo da universalização que preconiza a LNSB.

Como input, $\mathrm{x}_{1 k}$, utilizaremos o item FN015 - Despesas com Exploração - que se aproxima do conceito de Custo Operacional mensurada em R \$/ano cujos valores foram atualizados para 2013 utilizando o IGP-DI (Índice Geral de Preços - Disponibilidade Interna) da Fundação Getúlio Vargas.

A aplicação do método DEA será realizado por meio do pacote FEAR (Frontier Efficiency Analysis with $R$ ) do programa $\mathrm{R}^{8}$.

O pacote FEAR nos permitirá a aplicação bootstrap no DEA e no Malmquist e foi desenvolvido por Wilson (2008) e é disponibilizado em sua página pessoal ${ }^{9}$.

\section{RESULTADOS}

A apresentação dos resultados da análise estática precede os da análise dinâmica.

\subsection{Análise Estática}

A Tabela 1 apresenta os escores de eficiência calculados conjuntamente para as 216 DMUs, no período de 2006 a 2013. Observa-se que o nível de eficiência, em torno de 0,40 e 0,56, é muito baixo e embora se note uma melhora ao longo do período, essa não é contínua, o que evidencia uma grande margem para o aumento da eficiência técnica, ou seja, de acordo com a orientação do modelo significa a possibilidade de expansão dos outputs dada a quantidade de inputs Ademais se observa também um alto desvio padrão demonstrando grande heterogeneidade entre o desempenho das prestadoras.

\section{TABELA 1}

Estatísticas resumo dos escores - 2006-2013

\begin{tabular}{cccccccc}
\hline Ano & Mínimo & máximo & 1q quartil & Mediana & média & 3quartil & desvio padrão \\
\hline 2006 & 0.168 & 0.817 & 0.416 & 0.504 & 0.519 & 0.645 & 0.162 \\
2007 & 0.179 & 0.820 & 0.436 & 0.537 & 0.541 & 0.647 & 0.155 \\
2008 & 0.184 & 0.850 & 0.450 & 0.485 & 0.549 & 0.663 & 0.162 \\
2009 & 0.243 & 0.821 & 0.433 & 0.532 & 0.531 & 0.633 & 0.182 \\
2010 & 0.195 & 0.904 & 0.410 & 0.493 & 0.542 & 0.677 & 0.182 \\
2011 & 0.212 & 0.921 & 0.452 & 0.503 & 0.545 & 0.667 & 0.183 \\
2012 & 0.232 & 0.892 & 0.483 & 0.558 & 0.584 & 0.737 & 0.172 \\
\hline
\end{tabular}

Fonte: Elaboração dos autores.

O Gráfico 1 abaixo apresenta como esses escores estão correlacionados com a quantidade de economias de água que serve de aproximação para o tamanho da prestadora. Percebe-se com isso

\footnotetext{
${ }^{8} \mathrm{O}$ R é um software livre criado por Ross Ihaka e por Robert Gentleman da universidade de Auckland (Nova Zelândia) que permite a operação de métodos estatísticos e a formatação de gráficos e pode ser instalado e distribuído livremente.

${ }^{9}<\underline{\text { http://www.clemson.edu/economics/faculty/wilson/Software/FEAR/fear.html }>}$
} 
que, embora existam prestadoras eficientes de todos os tamanhos, há uma tendência da eficiência aumentar quanto maior for a quantidade de economias de água.

\section{GRÁFICO 1}

Quantidade de Economias de Água e Escore de Eficiência

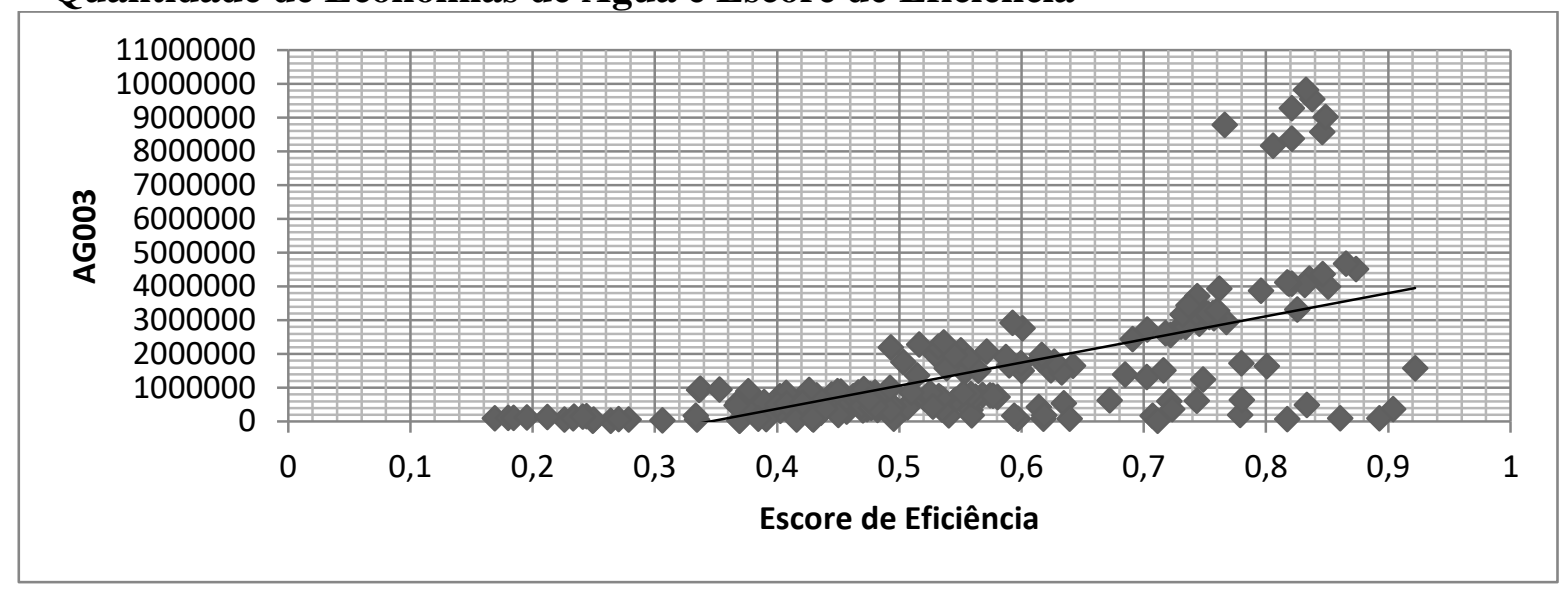

Fonte: Fonte: Elaboração dos autores.

\subsection{Análise Dinâmica}

A Tabela 2 apresenta os valores do Índice de Malmquist. Observa-se que a produtividade total dos fatores, em geral, cresceu durante o período analisado, exceto, para o período 2008-2009. Já entre 2012 e 2013 somente o índice medido pela média é também menor que um.

Observando a mediana, que é menos sensível a valores extremos do que a média, percebemos que o maior crescimento da produtividade total dos fatores ocorreu entre 2011-2012, cerca de 5\%, já o pior desempenho aconteceu entre 2008-2009, com uma queda de quase $9 \%$.

TABELA 2

Estatísticas da dinâmica da Produtividade Total dos Fatores, 2006-2013

\begin{tabular}{cccccccc}
\hline Ano inicial-final & mínimo & máximo & 1ㅇ quartil & Mediana & média & 3o quartil & desvio padrão \\
\hline $2006-2007$ & 0.829 & 1.488 & 0.980 & 1.033 & 1.042 & 1.095 & 0.122 \\
$2007-2008$ & 0.832 & 1.482 & 0.980 & 1.026 & 1.041 & 1.073 & 0.130 \\
$2008-2009$ & 0.766 & 1.765 & 0.858 & 0.913 & 0.941 & 0.964 & 0.178 \\
$2009-2010$ & 0.648 & 1.292 & 0.949 & 1.039 & 1.035 & 1.130 & 0.162 \\
$2010-2011$ & 0.703 & 2.256 & 0.931 & 1.001 & 1.064 & 1.118 & 0.272 \\
$2011-2012$ & 0.808 & 2.379 & 0.987 & 1.048 & 1.153 & 1.259 & 0.294 \\
$2012-2013$ & 0.437 & 1.436 & 0.946 & 1.019 & 0.970 & 1.041 & 0.173 \\
\hline
\end{tabular}

Fonte: Elaboração dos autores.

A Tabela 3 apresenta os efeitos da Mudança de Eficiência e, assim, é possível verificar que o componente de emparelhamento teve uma contribuição positiva entre 2006 e 2009, e destacadamente, no período final de 2012-2013. Já entre 2009 e 2012 a contribuição do "catch up" para a produtividade total dos fatores foi negativa. 
TABELA 3

Estatísticas da dinâmica da Mudança de Eficiência, 2006-2013

\begin{tabular}{cccccccc}
\hline Ano inicial-final & mínimo & Máximo & 1o quartil & mediana & média & 3o quartil & desvio padrão \\
\hline $2006-2007$ & 0.890 & 1.442 & 0.987 & 1.052 & 1.055 & 1.096 & 0.112 \\
$2007-2008$ & 0.792 & 1.448 & 1.000 & 1.041 & 1.069 & 1.123 & 0.124 \\
$2008-2009$ & 0.830 & 2.093 & 0.940 & 1.000 & 1.033 & 1.024 & 0.222 \\
$2009-2010$ & 0.539 & 1.117 & 0.842 & 0.926 & 0.909 & 1.000 & 0.139 \\
$2010-2011$ & 0.612 & 1.659 & 0.725 & 0.801 & 0.848 & 0.922 & 0.200 \\
$2011-2012$ & 0.501 & 1.235 & 0.621 & 0.779 & 0.789 & 0.977 & 0.192 \\
$2012-2013$ & 0.819 & 2.049 & 1.312 & 1.471 & 1.474 & 1.821 & 0.363 \\
\hline
\end{tabular}

Fonte: Elaboração dos autores.

Já a dinâmica da Mudança de Tecnologia apresenta uma evolução oposta à dinâmica da Mudança de Eficiência, conforme a Tabela 4, quando a contribuição é negativa no início e final do período analisado e positiva nos anos intermediários.

TABELA 4

Estatísticas resumo da Dinâmica da Mudança de Tecnologia 2006-2013

\begin{tabular}{cccccccc}
\hline Ano inicial-final & mínimo & máximo & 1ㅇ quartil & mediana & média & 3o quartil & desvio padrão \\
\hline $2006-2007$ & 0.888 & 1.101 & 0.949 & 0.993 & 0.987 & 1.012 & 0.051 \\
$2007-2008$ & 0.881 & 1.156 & 0.909 & 0.958 & 0.976 & 1.033 & 0.076 \\
$2008-2009$ & 0.802 & 1.005 & 0.904 & 0.923 & 0.914 & 0.926 & 0.042 \\
$2009-2010$ & 1.068 & 1.292 & 1.122 & 1.132 & 1.139 & 1.133 & 0.055 \\
$2010-2011$ & 0.991 & 1.457 & 1.167 & 1.346 & 1.260 & 1.349 & 0.142 \\
$2011-2012$ & 1.001 & 1.957 & 1.330 & 1.362 & 1.486 & 1.623 & 0.237 \\
$2012-2013$ & 0.512 & 1.112 & 0.565 & 0.668 & 0.680 & 0.714 & 0.141 \\
\hline
\end{tabular}

Fonte: Elaboração dos autores.

Para explorar melhor as estimativas, na tentativa de reduzir o impacto do período 2008-2009 na dinâmica da Produtividade Total dos Fatores, resolvemos dividir a análise em dois períodos, a saber: $2006-10$ e 2010-13. 


\section{TABELA 5}

Estatísticas Resumo do Índice Malmquist, 2006-10

\begin{tabular}{cccccccc}
\hline Índice de Malmquist & Mínimo & máximo & 1ำ quartil & mediana & média & 3o quartil & desvio padrão \\
\hline Eficiência & 0.753 & 1.595 & 0.938 & 1.000 & 1.030 & 1.082 & 0.166 \\
Tecnológica & 0.919 & 1.251 & 0.934 & 0.946 & 1.006 & 1.042 & 0.108 \\
Total & 0.705 & 1.639 & 0.899 & 1.006 & 1.037 & 1.151 & 0.203 \\
\hline
\end{tabular}

Fonte: Elaboração dos autores.

Conforme mostra a Tabela 5, as estatísticas de tendência central da Produtividade Total dos Fatores entre os anos de 2006 e 2010 foram em torno da unidade, indicando pouco avanço de eficiência no período.

Os resultados da Tabela 6 estimam para o período 2006-10 a quantidade percentual de prestadoras que se enquadram nos três casos: i) piorou maneira significativa: o intervalo de confiança encontrado apresenta os dois extremos menores do que a unidade; ii) melhorou de maneira significativa: o intervalo de confiança apresenta os dois extremos maiores que a unidade; e iii) não houve mudança significativa: o intervalo de confiança possui um extremo menor do que a unidade e outro maior.

Assim, verificamos para o período 2006 e 2010 que 40,7\% das prestadoras apresentaram melhora significativa, enquanto que $48,1 \%$ apresentaram piora. Já a mesma análise com os índices decompostos entre os efeitos mudança de eficiência e mudança tecnológica revela que a maioria das prestadoras, $70,3 \%$ e $62,9 \%$, respectivamente, não apresentaram mudança significativa.

TABELA 6

Mudança significativa de eficiência, 2006-2010.

\begin{tabular}{|c|c|c|c|}
\hline Índice de Malmquist & Melhora significativa & Piora significativa & Sem mudança significativa \\
\hline Eficiência & $22.2 \%$ & $7.4 \%$ & $70.3 \%$ \\
\hline Tecnologia & $11.1 \%$ & $25.9 \%$ & $62.9 \%$ \\
\hline Total & $40.7 \%$ & $48.1 \%$ & $11.1 \%$ \\
\hline
\end{tabular}

Fonte: Elaboração dos autores.

Para o período 2010-13 se observa na Tabela 7 um aumento significativo de eficiência somente atribuído ao componente tecnológico. Em torno de 55\% das unidades analisadas apresentaram melhoria significativa de eficiência, como mostra a Tabela 8. E também na Tabela 8 observamos que no efeito mudança tecnológica 59\% das unidades que apresentaram melhoria significativa e nenhuma teve piora significativa. Por outro lado, não há melhoria significativa nas unidades com o efeito emparelhamento enquanto quase $48 \%$ pioraram nesse componente. 
TABELA 7

Estatísticas Resumo do Índice Malmquist, 2010-13.

\begin{tabular}{cccccccc}
\hline $\begin{array}{c}\text { Índice de } \\
\text { Malmquist }\end{array}$ & mínimo & máximo & 1ำ quartil & mediana & média & 3qoartil & $\begin{array}{c}\text { desvio } \\
\text { padrão }\end{array}$ \\
\hline Eficiência & 0.538 & 1.659 & 0.820 & 0.905 & 0.925 & 0.994 & 0.198 \\
Tecnológica & 0.886 & 1.804 & 0.997 & 1.209 & 1.239 & 1.350 & 0.258 \\
Total & 0.702 & 2.994 & 0.968 & 1.067 & 1.146 & 1.163 & 0.412 \\
\hline
\end{tabular}

Fonte: Elaboração dos autores.

TABELA 8

Mudança significativa de eficiência, 2010-13.

\begin{tabular}{cccc}
\hline Malmquist & Melhora significativa & Piora significativa & Sem mudança significativa \\
\hline Eficiência & $0.0 \%$ & $48.1 \%$ & $51.8 \%$ \\
Tecnológica & $59.2 \%$ & $0.0 \%$ & $40.7 \%$ \\
Total & $55.5 \%$ & $29.6 \%$ & $14.8 \%$ \\
\hline
\end{tabular}

No o período analisado, ou seja, entre 2006 e 2013, podemos perceber na Tabela 9 que as estatísticas resumo demonstram um avanço na produtividade total dos fatores no período. Esse aumento, como entre 2010 e 2013, é explicado pela mudança tecnológica, já que a mudança de eficiência apresentou média e mediana menores do que a unidade.

TABELA 9

Estatísticas Resumo do Índice Malmquist, 2006-2013.

\begin{tabular}{cccccccc}
\hline Índice de Malmquist & mínimo & máximo & 1ำquartil & mediana & média & 3qo quartil & desvio padrão \\
\hline Eficiência & 0.487 & 1.698 & 0.818 & 0.894 & 0.952 & 1.008 & 0.245 \\
Tecnológica & 0.939 & 1.969 & 1.106 & 1.220 & 1.248 & 1.369 & 0.221 \\
Total & 0.802 & 2.841 & 0.979 & 1.067 & 1.194 & 1.150 & 0.451 \\
\hline
\end{tabular}

Fonte: Elaboração dos autores.

A análise da mudança significativa de cada prestadora, Tabela 10, também indica avanço entre 2006 e 2013, principalmente na mudança tecnológica, onde mais de $51 \%$ das prestadoras apresenta melhora significativa e nenhuma piora significativamente. 
TABELA 10

Mudança significativa de eficiência, 2006-2013.

\begin{tabular}{cccc}
\hline Malmquist & Melhora significativa & Piora significativa & Sem mudança significativa \\
\hline Eficiência & $7.4 \%$ & $33.3 \%$ & $59.2 \%$ \\
Tecnológica & $51.8 \%$ & $0.0 \%$ & $48.1 \%$ \\
Total & $40.7 \%$ & $22.2 \%$ & $37.0 \%$ \\
\hline
\end{tabular}

Fonte: Elaboração dos autores.

\section{CONCLUSÃO}

Com o objetivo de analisar a eficiência na prestação dos serviços de água e esgoto depois da LNSB, selecionamos as maiores prestadoras de cada Estado entre os anos de 2006 e 2013 e aplicamos o método DEA em conjunto com o bootstrap para a análise estática da eficiência técnica e o Índice de Malmquist, também em conjunto com o bootstrap, para uma análise dinâmica.

$\mathrm{Na}$ análise estática percebemos que os escores no último ano permanecem baixos e a análise da trajetória da eficiência por meio das medidas de tendência central foi inconclusiva. Assim, melhorando a eficiência técnica existe a possibilidade de aumentar os outputs sem a necessidade de elevar os custos operacionais, o que poderia ocorrer diminuindo a heterogeneidade entre as performances das prestadoras.

Já a análise da dinâmica realizada ano a ano com a utilização do Índice de Malmquist nos informa que, em cinco dos sete anos, os valores da média e da mediana apontam para o avanço da produtividade total dos fatores. Apenas entre os anos de 2008 e 2009 verificamos média e mediana menores do que unidade. Por outro lado, entre os anos de 2012 e 2013 observamos indicações opostas das duas medidas de tendência central observadas. Nessa mesma análise percebemos que enquanto a mudança de eficiência foi predominante entre os anos de 2006 e 2009 e no período entre 2012 e 2013, a mudança de tecnologia foi maior entre 2009 e 2012.

Ao dividirmos a análise entre dois períodos maiores: 2006-2010 e 2010-2013, percebemos que, entre 2006 e 2010, apesar das medidas de tendência central apontarem para um avanço no período liderado pelo "efeito emparelhamento", uma análise das mudanças significativas revelam que mais prestadoras diminuíram sua produtividade total dos fatores do que aumentaram. E também que, em relação às mudanças de tecnologia e de eficiência, a maioria das prestadoras não apresentou mudança significativa.

A situação se apresenta de maneira mais favorável entre o período de 2010 e 2013. Isso porque, tanto a análise das medidas de tendência central, quanto a análise das mudanças significativas apontam para um avanço na eficiência no período e que essa melhora foi liderada pela mudança de tecnologia.

A análise de todo o período de 2006 a 2013 apontou que o avanço na produtividade total dos fatores durante o período é explicado pela mudança de tecnologia. Assim, verificamos que a expansão da fronteira foi mais relevante para o aumento da produtividade total dos fatores do que o efeito emparelhamento e, portanto, indica-se que a introdução de novos processos e técnicas foram mais importantes do que a simples aplicação da atual tecnologia pelas prestadoras atrasadas.

Dessa forma, nossos resultados encontraram uma melhoria de eficiência mais significativa que Carvalho (2014) que analisam período similar. Já em comparação ao trabalho de Seroa e Moreira (2006) que analisa um período anterior até 2004, a diferença dos nossos resultados seria a predominância da mudança tecnológica sobre o efeito de emparelhamento.

Contudo, observamos um nível de eficiência relativamente baixo e heterogêneo e, portanto, o avanço no período não se mostrou suficiente para mudar a realidade dos serviços de água e esgotos no país.

Por fim, não temos como atribuir que essa quebra estrutural no ano de 2010, quando a melhoria de eficiência é mais acentuada, se deve entrada em vigência da LNSB. Isto porque ainda 
não se mensurou o grau e a incidência territorial da sua implementação e, de acordo com a observação de especialistas, essa tem sido lenta e gradual ${ }^{10}$, em particular com a atuação de agência reguladora.

Outra conjectura seria que essa quebra resultaria dos novos investimentos fomentados pelo Programa de Aceleração do Crescimento (PAC), mas as características deles não são observáveis e eles não representariam, a princípio, um incremento substancial no estoque atual de estações e facilidades de saneamento.

De qualquer forma, nosso estudo apenas retratou a evolução da eficiência no setor. Estudos futuros poderão analisar como fatores de mercado ou regulatórios criaram ou não incentivos para essa trajetória verificada. 


\section{REFERÊNCIAS}

ABBOTT, M.; COHEN, B.; WANG, W. C. The performance of the urban water and wastewater sectors in Australia. Utilities Policy, v. 20, n. 1, p. 52-63, 2012.

BANKER, R. D.; CHARNES, A.; COOPER, W. W. Some models for estimating technical and scale inefficiencies in data envelopment analysis, Management Science, v. 30, p. 1078-1092, 1984.

BARBOSA, A. Pode a regulação econômica melhorar o desempenho econômico-financeiro e a universalização dos serviços de águas e esgotos no Brasil? Brasília: SEAE, 2012. 67 p.

BOGETOFT, P.; OTTO, L. Benchmarking with DEA, SFA and R. New York: Springer, 2011.

BRASIL. Lei $\mathrm{n}^{\circ} 11.445$, de 5 de janeiro de 2007. Estabelece diretrizes nacionais para $o$ saneamento básico; altera as Leis $n^{\circ}$ 6.766, de 19 de dezembro de 1979, 8.036, de 11 de maio de 1990, 8.666, de 21 de junho de 1993, 8.987, de 13 de fevereiro de 1995; revoga a Lei $n^{\circ} 6.528$, de 11 de maio de 1978; e dá outras providências. Disponível em: <http://www.planalto.gov.br/ccivil_03/_ato2007-2010/2007/lei/111445.htm>. Acesso em: 30 out. 2014.

BRASIL. Ministério da saúde. Programa de saneamento básico. Brasília: ed. MS, 2002.

BYRNES, J. et al. The relative economic efficiency of urban water utilities in regional New South Wales and Victoria. Resource and Energy Economics, v. 32, n. 3, p. 439-455, 2010.

CARMO, C. M. do; TAVORA JUNIOR, J. L. Avaliação da eficiência técnica das empresas de saneamento brasileiras utilizando a metodologia DEA. In: Encontro Nacional de Economia da ANPEC, n. 31, Porto Seguro, 2003. Disponível em: <http://www.anpec.org.br/encontro2003/artigos/D32.pdf >. Acesso em: 05 mar. 2016.

CARVALHO, A. E. C. Caminhos para a universalização dos serviços de água e esgotos no Brasil: a atuação das entidades reguladoras para indução da eficiência dos prestadores de serviços. Dissertação (Mestrado) - Curso de Gestão Pública, Universidade Federal do Rio Grande do Norte (PPGP/UFRN), Natal, 2014, 140 p. $\quad$ Disponível em: <http://repositorio.ufrn.br/jspui/handle/123456789/16905>. Acesso em: 03 abr. 2016.

CHARNES, A.; COOPER, W. W.; RHODES, E. Measuring the efficiency of decision making units. European Journal of Operational Research, v. 2, n. 4, p. 429-444, 1978.

ESTACHE A.; GARSOUS G.; SEROA DA MOTTA R. Shared Mandates, Moral Hazard, and Political (Mis)alignment in a Decentralized Economy. World Development, v. 83, p. 98-110, 2016.

GRIGOLIN, R. Setor de água e saneamento no Brasil: regulamentação e eficiência. São Paulo: FGV, 2007. Dissertação (Mestrado), Escola de Economia de São Paulo, Fundação Getúlio Vargas, São Paulo, 2007, 64 p.

INSTITUTO BRASILEIRO DE GEOGRAFIA E ESTATÍSTICA - IBGE. (Ed.). Síntese de Indicadores Sociais: uma análise das condições de vida da população brasileira 2014. Rio de Janeiro: $\quad$ IBGE, 2014. Disponível em: 〈http://biblioteca.ibge.gov.br/visualizacao/livros/liv91983.pdf>. Acesso em: 27 fev. 2016.

LIN C.; BERG S. V. Incorporating service quality into yardstick regulation: an application to the Peru water sector. Review of Industrial Organization, v. 32, n.1, p. 53-75, 2008. 
MARINHO, A.; FAÇANHA, L. O. Programas sociais: efetividade, eficiência e eficácia como dimensões operacionais da avaliação. Texto para Discussão 787, IPEA, Rio de Janeiro, 22 p., 2001.

MENDONÇA, M. J.; SEROA DA MOTTA, R. Saúde e saneamento no Brasil, Planejamento $e$ Políticas Públicas 30, p. 15-30, 2007.

BRASIL, MINISTÉRIO DAS CIDADES. Secretaria Nacional de Saneamento Ambiental. Sistema nacional de informações sobre saneamento: diagnóstico dos serviços de água e esgotos - 2013. Brasília: MCIDADES/SNSA, 2015. 58 p.

PORTELA, M. et al. Productivity change in the water industry in England and Wales: application of the meta-Malmquist index. Journal of the Operational Research Society, v. 62, n. 12, p. 2173$2188,2011$.

SALLES, M. J. Política Nacional de Saneamento: percorrendo caminhos em busca da universalização. 2008. 176 p., Tese (Doutorado) - Curso de Ciências na área de Saúde Pública, Escola Nacional de Saúde Pública - ENSP, Rio de Janeiro, 2008. Disponível em: <http://www.arca.fiocruz.br/handle/icict/2605>. Acesso em: 27 fev. 2016.

SAMPAIO, B.; SAMPAIO, Y. Influências políticas na eficiência de empresas de saneamento brasileiras. Economia Aplicada, São Paulo, v. 11, n. 3, p. 369-386, jul.-set., 2007.

SATO, J. M. Utilização da análise envoltória de dados (DEA) no estudo de eficiência do setor de saneamento. Brasília: UCB, 2011. 43 p. Dissertação (Mestrado), Programa de Pós-graduação em Economia Regional, Universidade Católica de Brasília, Brasília, 2011.

SEROA DA MOTTA, R.; MOREIRA, A. Efficiency and regulation in the sanitation sector in Brazil. Utilities Policy. v. 14, p. 185-195, 2006.

SEROA DA MOTTA, R. A ausência de regulação econômica em saneamento no Brasil in SALGADO, L. H.; SEROA DA MOTTA, R. (eds.) Marcos Regulatórios no Brasil: $O$ que foi feito e o que falta fazer, IPEA, Rio de Janeiro, 2005.

SIMAR, L.; WILSON, P. Sensitivity analysis of efficiency scores: how to bootstrap in nonparametric frontier models. Management science, v. 44, n. 1, p. 49-61, 1998.

SIMAR, L; WILSON, P.W. Theory and methodology: estimating and bootstrapping Malmquist index. European Journal of operational research, v. 115, p. 459-471, 1999.

TUPPER, H. C.; RESENDE, M. Efficiency and regulatory issues in the Brazilian water and sewage sector: an empirical study. Utilities Policy, n. 12, p. 29-40, 2004.

WILSON, P. W. FEAR 1.0: a software package for frontier efficiency analysis with R. Socioeconomic planning sciences, v. 42, n. 4, p. 247-254, 2008. 